(C) 2019 IEEE. Personal use of this material is permitted. Permission from IEEE must be obtained for all other uses, in any current or future media, including reprinting/republishing this material for advertising or promotional purposes, creating new collective works, for resale or redistribution to servers or lists, or reuse of any copyrighted component of this work in other works.

DOI: 10.1109/CLEOE-EQEC.2019.8872483 


\title{
VCSEL Technologies for High-capacity Dense WDM Networks
}

\author{
Mariangela Rapisarda ${ }^{1}$, Alberto Gatto ${ }^{1}$, Paolo Martelli $^{1}$, Paola Parolari $^{1}$, Christian Neumeyr ${ }^{2}$, \\ Michela Svaluto Moreolo ${ }^{3}$, Josep M. Fabrega ${ }^{3}$, Laia Nadal ${ }^{3}$, and Pierpaolo Boffi ${ }^{1}$ \\ 1. Politecnico di Milano, Dip. Elettronica Informazione e Bioingegneria, P.zza Leonardo da vinci 32, 20133, Milano Italy \\ 2. Vertilas GmbH, Daimlerstr. 11d, D-85748 Garching, Germany \\ 3. Centre Tecnològic de Telecomunicacions de Catalunya (CTTC/CERCA), Av. C. F. Gauss 7, 08860 Castelldefels (Barcelona), Spain
}

Long-wavelength vertical cavity surface emitting laser (VCSEL) technologies can represent an alternative solution for the development of transmitters with reduced cost, power consumption and footprint for very-high capacity metropolitan area systems. Exploiting direct modulation (DM) and advanced modulation formats, such as discrete multitone (DMT), and aggregating multiple DM-VCSELs emitting in the C-band with dense wavelength division multiplexers (WDM) in SOI chips allows to achieve multi-Tb/s transmitter module with $25 \mathrm{GHz}$ granularity [1].

The interplay between the frequency chirp induced by VCSELs DM and the tight filtering of network WDM multiplexers plays a crucial role in the transmission performance. In this paper, we compare the adoption of two different long-wavelength InP VCSEL technologies: high-bandwidth short-cavity devices and MEMS-based widely-tuneable VCSELs [1]. We evaluate their performance in case of DM employing DMT modulation, providing more than $50-\mathrm{Gb} / \mathrm{s}$ capacity per wavelength; coherent detection (COH-D) is used to target hundreds of kilometres distances thanks to DSP chromatic dispersion (CD) compensation.

At first, we performed chirp measurements on a short-cavity 20-GHz long-wavelength VCSEL emitting at $1533.4 \mathrm{~nm}$, and on an 8-GHz MEMS-based VCSEL tuneable over about $70 \mathrm{~nm}$ in the $\mathrm{C}$ band [2], see Fig. $1 \mathrm{a}$ ).

These parameters were used to evaluate the transmission performance as a function of the optical signal to noise ratio (OSNR) and of the number of crossed nodes (i.e. number of WDM filters) employing a 25-GHz channel spacing WSS, with 21-GHz FWHM. The VCSEL modulation frequency bandwidth was set to $18 \mathrm{GHz}$ in both cases to specifically evaluate the chirp impact. We considered both dual sideband (DSB) and single sideband (SSB) DMT modulations with less than $25 \mathrm{GHz}$ optical spectrum, composed by 256 sub-carriers and a cyclic prefix of about $2.1 \%$ of the symbol length; the signal electrical bandwidths were $10 \mathrm{GHz}$ and $20 \mathrm{GHz}$ respectively.

(a)

\begin{tabular}{|c|c|c|}
\hline VCSEL & $\begin{array}{c}\boldsymbol{\alpha} \text { linewidth enh. } \\
\text { factor }\end{array}$ & к adiabatic \\
\hline Tuneable & 2.75 & $8.3 \cdot 10^{12}$ \\
\hline $\begin{array}{c}\text { Short- } \\
\text { cavity }\end{array}$ & 3.7 & $1.52 \cdot 10^{13}$ \\
\hline
\end{tabular}

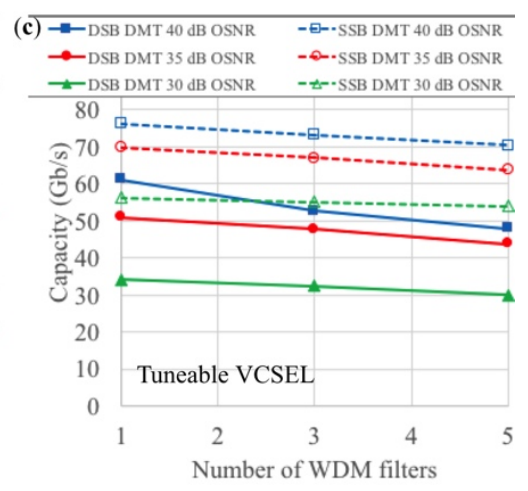

Fig. 1 a) Measured chirp coefficients. b)-c) Transmission capacities vs number of crossed WDM filters for: 40-dB OSNR (blue square), 35-dB OSNR (red circle), 30-dB OSNR (green triangle), and DSB DMT modulation (continuous line full symbols), SSB DMT modulation (dashed line open symbol) b) short-cavity VCSEL . c) tuneable VCSEL.

Fig. $1 \mathrm{~b}$ ) and c) show that lower chirp (tuneable) devices support much higher capacities for a higher number of crossed nodes, in particular for DSB modulation; however, SSB outperforms DSB modulation due to a higher spectral efficiency. $50 \mathrm{~Gb} / \mathrm{s}$ capacity per wavelength can be achieved in case of short cavity technology only for SSB DMT modulation and the evaluated OSNR range guarantees this target for more than $260 \mathrm{~km}$ SSMF transmission. However, if devices characterized by chirp parameters as the tuneable VCSEL would be available in combination with short-cavity-like modulation bandwidths, even the DSB DMT modulation would allow a $50 \mathrm{~Gb} / \mathrm{s}$ capacity for $40 \mathrm{~dB}$ OSNR and up to $3 \mathrm{WDM}$ filters with a maximum reach of $200 \mathrm{~km}$.

This work is funded by the EU H2020 programme, PASSION project GA 780326

\section{References}

[1] M. Svaluto Moreolo et al., "VCSEL-based sliceable bandwidth/bitrate variable transceivers," SPIE-PWO, San Francisco, CA (USA), Feb. 2019.

[2] M. Rapisarda, et al. "Impact of Chirp in High-Capacity Optical Metro Networks Employing Directly-Modulated VCSELs," Photonics 5 (4), 51 (2018). 